\title{
Tau pathology and relative cerebral blood flow are independently associated with cognition in Alzheimer's disease
}

\author{
Denise Visser $^{1}$ (D) $\cdot$ Emma E. Wolters $^{1,2} \cdot$ Sander C. J. Verfaillie $^{1} \cdot$ Emma M. Coomans $^{1} \cdot$ Tessa Timmers $^{1,2} \cdot$ \\ Hayel Tuncel ${ }^{1}$ - Juhan Reimand ${ }^{2}$ - Ronald Boellaard ${ }^{1}$ - Albert D. Windhorst ${ }^{1}$ • Philip Scheltens ${ }^{2} \cdot$ Wiesje M. van \\ der Flier ${ }^{2,3} \cdot$ Rik Ossenkoppele $^{2,4} \cdot$ Bart N. M. van Berckel $^{1}$
}

Received: 23 October 2019 / Accepted: 16 April 2020 / Published online: 27 May 2020

(C) The Author(s) 2020

\begin{abstract}
Purpose We aimed to investigate associations between tau pathology and relative cerebral blood flow (rCBF), and their relationship with cognition in Alzheimer's disease (AD), by using a single dynamic $\left[{ }^{18} \mathrm{~F}\right]$ flortaucipir positron emission tomography (PET) scan.

Methods Seventy-one subjects with AD (66 \pm 8 years, mini-mental state examination (MMSE) $23 \pm 4)$ underwent a dynamic 130-min $\left[{ }^{18} \mathrm{~F}\right]$ flortaucipir PET scan. Cognitive assessment consisted of composite scores of four cognitive domains. For tau pathology and $\mathrm{rCBF}$, receptor parametric mapping (cerebellar gray matter reference region) was used to create uncorrected and partial volume-corrected parametric images of non-displaceable binding potential $\left(\mathrm{BP}_{\mathrm{ND}}\right)$ and $R_{l}$, respectively. (Voxel-wise) linear regressions were used to investigate associations between $\mathrm{BP}_{\mathrm{ND}}$ and/or $R_{l}$ and cognition.

Results Higher $\left[{ }^{18} \mathrm{~F}\right]$ flortaucipir $\mathrm{BP}_{\mathrm{ND}}$ was associated with lower $R_{l}$ in the lateral temporal, parietal and occipital regions. Higher medial temporal $\mathrm{BP}_{\mathrm{ND}}$ was associated with worse memory, and higher lateral temporal $\mathrm{BP}_{\mathrm{ND}}$ with worse executive functioning and language. Higher parietal $\mathrm{BP}_{\mathrm{ND}}$ was associated with worse executive functioning, language and attention, and higher occipital $\mathrm{BP}_{\mathrm{ND}}$ with lower cognitive scores across all domains. Higher frontal $\mathrm{BP}_{\mathrm{ND}}$ was associated with worse executive function and attention. For $\left[{ }^{18} \mathrm{~F}\right]$ flortaucipir $R_{l}$, lower values in the lateral temporal and parietal ROIs were associated with worse executive functioning, language and attention, and lower occipital $R_{l}$ with lower language and attention scores. When $\left[{ }^{18} \mathrm{~F}\right]$ flortaucipir $\mathrm{BP}_{\mathrm{ND}}$ and $R_{l}$ were modelled simultaneously, associations between lower $R_{l}$ in the lateral temporal ROI and worse attention remained, as well as for lower parietal $R_{l}$ and worse executive functioning and attention.

Conclusion Tau pathology was associated with locally reduced rCBF. Tau pathology and low rCBF were both independently associated with worse cognitive performance. For tau pathology, these associations spanned widespread neocortex, while for $\mathrm{rCBF}$, independent associations were restricted to lateral temporal and parietal regions and the executive functioning and attention domains. These findings indicate that each biomarker may independently contribute to cognitive impairment in AD.
\end{abstract}

Keywords $\left[{ }^{18} \mathrm{~F}\right]$ flortaucipir PET $\cdot$ Relative cerebral blood flow $\cdot$ Tau $\cdot$ Cognition $\cdot$ Alzheimer's disease

Denise Visser and Emma E. Wolters contributed equally to this work.

This article is part of the Topical Collection on Neurology.

Electronic supplementary material The online version of this article (https://doi.org/10.1007/s00259-020-04831-w) contains supplementary material, which is available to authorized users.

Denise Visser

d.visser2@amsterdamumc.nl

1 Department of Radiology \& Nuclear Medicine, Amsterdam Neuroscience, Vrije Universiteit Amsterdam, Amsterdam UMC, Amsterdam, The Netherlands
2 Alzheimer Center Amsterdam, Department of Neurology, Amsterdam Neuroscience, Vrije Universiteit Amsterdam, Amsterdam UMC, Amsterdam, The Netherlands

3 Department of Epidemiology and Biostatistics, Vrije Universiteit Amsterdam, Amsterdam UMC, Amsterdam, The Netherlands

4 Clinical Memory Research Unit, Lund University, Lund, Sweden 


\section{Introduction}

$\left[{ }^{18} \mathrm{~F}\right]$ Flortaucipir is the most widely studied PET tracer to date for detecting AD-specific tau pathology [1]. Most studies with $\left[{ }^{18} \mathrm{~F}\right]$ flortaucipir used static scan protocols, which allow semiquantitative estimates such as the standardized uptake value ratio (SUVR) [1]. Advantages of static over dynamic scanning protocols include the relatively short scan duration and computational simplicity which facilitates clinical applicability [2]. On the other hand, dynamic acquisition allows optimal quantitative accuracy and additionally enables computation of parametric images of tracer delivery, which can be interpreted as a proxy of relative tracer flow or relative cerebral blood flow (rCBF) (i.e. $R_{l}$ ) [2-9]. $R_{1}$ represents the ratio between the rate constant for ligand transfer from blood to tissue $\left(K_{l}\right)$ in the tissue of interest and the reference region [4-8], which is strongly correlated with metabolic activity derived from $\left[{ }^{18} \mathrm{~F}\right] \mathrm{FDG}$ PET $[4,5,9]$. A dynamic $\left[{ }^{18} \mathrm{~F}\right]$ flortaucipir PET scan may thus not only provide accurate information on (regional) quantification of tau pathology, but also yields information on rCBF.

Previous studies demonstrated that high levels of regional tau pathology [10-12], as well as low levels of rCBF (as measured with $\left[{ }^{18} \mathrm{~F}\right]$ FDG PET or MRI) $[9,13]$, correlate with cognitive impairment in various domains. However, $\mathrm{rCBF}$ has not been investigated yet using $\left[{ }^{18} \mathrm{~F}\right]$ flortaucipir $R_{1}$. Investigating tau pathology and $\mathrm{rCBF}$ simultaneously by using dynamic $\left[{ }^{18} \mathrm{~F}\right]$ flortaucipir PET might yield valuable information, since both pathophysiological mechanisms may contribute to cognitive impairment in $\mathrm{AD}$.

The aims of this study are to investigate the (regional) association between tau pathology and $\mathrm{rCBF}$, and their (independent) associations with cognitive functioning in patients with $\mathrm{AD}$.

\section{Methods}

\section{Recruitment of participants}

Patients were recruited from the Amsterdam Dementia Cohort of the Alzheimer Center Amsterdam [14]. All subjects underwent a standardized dementia screening, including medical and neurological examination, informant-based history, assessment of vital functions, screening laboratory tests, neuropsychological evaluation, MRI, lumbar puncture and/or amyloid- $\beta$ positron emission tomography (PET), after which diagnoses were determined in a multidisciplinary consensus meeting [14]. For this study, patients with a diagnosis of Alzheimer's disease (AD) dementia [15] or mild cognitive impairment (MCI) due to AD [16] were included. For all subjects, AD biomarkers in cerebrospinal fluid (CSF) and/or A $\beta$ PET were abnormal (CSF A $\beta 42<813 \mathrm{pg} / \mathrm{mL}[17]$ and/or abnormal A $\beta$ PET (on visual read)). According to the NIAAA Research Framework [18], all subjects are considered in the AD pathophysiological continuum. Subjects were excluded if they had severe traumatic brain injury, abnormalities on MRI likely to interfere with segmentation of tau PET and participation in drug trial with a tau or $A \beta$-targeting agent.

All procedures were in accordance with the ethical standards of the Medical Ethics Review Committee of the Amsterdam UMC VU Medical Center and with the 1964 Helsinki Declaration and its later amendments or comparable ethical standards. Informed consent was obtained from all individual participants included in the study.

\section{Image acquisition}

All participants underwent a single dynamic $\left[{ }^{18} \mathrm{~F}\right]$ flortaucipir PET scan at the Amsterdam UMC VU Medical Center on an Ingenuity TF PET-CT scanner (Philips Medical Systems, Best, The Netherlands) within 1 year from their neuropsychological examination. $\left[{ }^{18} \mathrm{~F}\right]$ Flortaucipir was synthesized at the Amsterdam UMC VU Medical Center, using a protocol described in detail previously [19]. The scan protocol started with a low-dose $\mathrm{CT}$ for attenuation correction, followed by a $234 \pm 14 \mathrm{MBq}\left[{ }^{18} \mathrm{~F}\right]$ flortaucipir bolus injection (injected mass $1 \pm 1 \mu \mathrm{g})$. Simultaneously with tracer injection, a 60-min dynamic emission scan was initiated. After a 20 -min break and following a second low-dose $\mathrm{CT}$ for attenuation correction, an additional dynamic emission scan was performed during the interval 80-130 min post-injection. During scanning, head movements were restricted by a head holder with band and head position was regularly checked. PET scans were reconstructed using a matrix size of $128 \times 128 \times 90$ and a final voxel size of $2 \times 2 \times 2 \mathrm{~mm}^{3}$. All standard corrections for dead time, decay, attenuation, randoms and scatter were performed. Both scan sessions were co-registered into a single dataset of 29 frames $(1 \times 15,3 \times 5,3 \times 10,4 \times 60,2 \times 150,2 \times 300,4 \times$ 600 and $10 \times 300 \mathrm{~s}$ ), in which the last 10 frames belonged to the second PET session.

In addition, all subjects underwent structural MRI on a 3.0 Tesla (3 T) Philips medical systems' Ingenuity TF PET-MRI. The protocol included an isotropic structural 3D T1-weighted image using a sagittal turbo gradient-echo sequence $\left(1.00 \mathrm{~mm}^{3}\right.$ isotropic voxels, repetition time $=7.9 \mathrm{~ms}$, echo time $=4.5 \mathrm{~ms}$, and flip angle $=8^{\circ}$ ), and a 3D fluid-attenuated inversion recovery (FLAIR) image $(1.04 \times 1.04 \times 1.12 \mathrm{~mm}$ voxels, repetition time $=4800 \mathrm{~ms}$, echo time $=278.8 \mathrm{~ms}$, flip angle $90^{\circ}$ ).

\section{PET and MR analyses}

Using Vinci software (Max Plank Institute, Cologne, Germany), T1-weighted MR images were co-registered to their individual PET scans in native space. To delineate 
cortical gray matter regions-of-interest (ROIs) on the coregistered MR images, the Hammers template [20] incorporated in PVElab software was used (which uses the default settings of SPM to define gray matter). To generate voxelwise parametric images of non-displaceable binding potential $\left(\mathrm{BP}_{\mathrm{ND}}\right)$ and $R_{1}$, receptor parametric mapping (RPM) [21] with cerebellar gray matter as reference region was applied to the dynamic 130 min PET data [22]. Our group previously demonstrated that, when compared to full kinetic modelling, RPM is the most optimal simplified parametric method for $\left[{ }^{18} \mathrm{~F}\right]$ flortaucipir [23] with excellent test-retest repeatability [24]. PET images were partial volume-corrected using Van Cittert iterative deconvolution methods (IDM), combined with highly constrained back-projection (HYPR) [25]. A moving frame composite image was used for HYPR to better sustain the temporal information while denoising [26]. Uncorrected data are presented throughout the paper and partial volume-corrected data are presented in the Supplementary material.

For voxel-wise analyses, using Statistical Parametric Mapping (SPM) version 12 software (Wellcome Trust Center for Neuroimaging, University College London, UK), we warped all native space parametric $\mathrm{BP}_{\mathrm{ND}}$ and $R_{l}$ images to Montreal Neurological Institute (MNI152) space, by using the transformation matrixes derived from warping the coregistered T1-weighted MRI scans to MNI space. Warped images underwent quality control for transformation errors.

For regional analyses, the following bilateral ROIs were created a priori based on the Hammers atlas [20] (in subject space): medial temporal (hippocampus, parahippocampal and ambient gyri, anterior temporal lobe medial part), lateral temporal (superior temporal gyrus, middle and inferior temporal gyri), parietal (inferolateral remainder of parietal lobe, superior parietal gyrus, gyrus cinguli posterior part), occipital (cuneus, lingual gyrus, lateral remainder of occipital lobe) and frontal (middle frontal gyrus, orbitofrontal gyri, superior frontal gyrus) regions.

As a measure of vascular pathology, white matter hyperintensities (WMHs) were visually rated by an experienced rater on subjects' FLAIR image using the Fazekas scale, with scores ranging from 0 to 3 [27].

\section{Cognition}

Cognitive domain scores were created by averaging Ztransformed test-scores (based on the current sample) of corresponding tests for memory (Immediate Recall of the Dutch version of the RAVLT, Delayed Recall of the Dutch version of the RAVLT and Visual Association Test-A), executive functioning (Stroop Colour Word test III, Phonemic Verbal Fluency (D-A-T), Digit Span Backwards and Trail Making Test (TMT)-B), language (Category Fluency Animals and Visual Association Test-Naming) and attention (TMT-A,
Stroop Colour Word test I and II and the Digit Span Forward) [28]. Tests on which lower scores indicated better performance (TMT-A and -B, Stroop Colour Word test I, II and III) were inverted. Domain scores were only calculated if two or more tests within a domain were available.

\section{Statistical analyses}

To assess the correlations between $\left[{ }^{18} \mathrm{~F}\right]$ flortaucipir $\mathrm{BP}_{\mathrm{ND}}$ and $R_{l}$, with age, sex, education and Fazekas score, a correlation matrix was created using Spearman correlations. A $p$ value below 0.05 was considered statistically significant.

To examine the regional associations between $\left[{ }^{18} \mathrm{~F}\right]$ flortaucipir $\mathrm{BP}_{\mathrm{ND}}$ and $R_{l}$, linear regression analyses, adjusted for age and sex, were performed. To assess the contribution of white matter damage in these associations, analyses were additionally adjusted for Fazekas score.

To assess voxel-wise associations between $\left[{ }^{18} \mathrm{~F}\right]$ flortaucipir $\mathrm{BP}_{\mathrm{ND}}$ or $R_{l}$ and cognition, voxel-wise regression analyses using SPM12 were performed. Analyses were adjusted for age, sex and education. A $p$ value below 0.001 (uncorrected) was considered statistically significant for voxel-wise analyses. Additionally, a more conservative family-wise error (FWE) correction at $p<0.05$ was applied.

To investigate regional associations between $\left[{ }^{18} \mathrm{~F}\right]$ flortaucipir $\mathrm{BP}_{\mathrm{ND}}$ or $R_{l}$ and cognition (dependent variables), linear regression analyses, adjusted for age, sex and education (model 1), were used. Subsequently, we entered $\left[{ }^{18} \mathrm{~F}\right.$ ]flortaucipir $\mathrm{BP}_{\mathrm{ND}}$ and $R_{1}$ simultaneously in the model to assess their independent associations with cognition (model 2).

For all regional analyses, we report the level of significance both with and without correction for multiple comparisons using the Benjamini-Hochberg procedure with a false discovery rate (FDR) Q value of 5\%. A $p$ value below 0.05 was considered statistically significant. All regional and voxelwise analyses were repeated with partial volume-corrected data.

\section{Results}

\section{Participants}

A total of 71 subjects (MCI due to AD: $n=10$, and AD dementia: $n=61$ ) with a mean age of $66 \pm 8$ years and MMSE score of $23 \pm 4$ were included (Table 1). By study design, all subjects had abnormal amyloid biomarkers. $\left[{ }^{18} \mathrm{~F}\right]$ Flortaucipir $\mathrm{BP}_{\mathrm{ND}}$ values were highest in parietal $(0.55 \pm 0.43)$ regions and $R_{l}$ values were lowest in medial temporal regions $(0.68 \pm$ 0.06) (Table 1). $\left[{ }^{18} \mathrm{~F}\right]$ Flortaucipir $\mathrm{BP}_{\mathrm{ND}}$ and/or $R_{l}$ showed statistically significant correlations with age and education (Table 2), but not with sex. 
Table 1 Overview of demographics, $\left[{ }^{18} \mathrm{~F}\right]$ flortaucipir $\mathrm{BP}_{\mathrm{ND}}$ and $R_{l}$

$$
N=71
$$

\begin{tabular}{ll} 
Diagnosis & \\
MCI due to AD $(n)$ & 10 \\
AD dementia $(n)$ & 61 \\
Age (years) & $66(8)$ \\
Sex (female/male) & $36 / 35$ \\
Education (Dutch Verhage scale) & $6[3-7]$ \\
Fazekas score & $1[0-3]$ \\
MMSE & $23(4)$ \\
{$\left[{ }^{18}\right.$ F $]$ flortaucipir BP } & \\
Medial temporal & \\
Lateral temporal & $0.25 \pm 0.15[-0.11-0.59]$ \\
Parietal & $0.48 \pm 0.30[-0.12-1.29]$ \\
Occipital & $0.55 \pm 0.43[-0.16-1.83]$ \\
Frontal & $0.45 \pm 0.40[-0.05-1.82]$ \\
{$\left[{ }^{18}\right.$ F $]$ flortaucipir $R_{l}$} & $0.26 \pm 0.27[-0.22-0.94]$ \\
Medial temporal & \\
Lateral temporal & $0.68 \pm 0.06[0.57-0.86]$ \\
Parietal & $0.86 \pm 0.08[0.70-1.13]$ \\
Occipital & $0.87 \pm 0.11[0.61-1.30]$ \\
Frontal & $0.98 \pm 0.10[0.74-1.34]$ \\
\hline
\end{tabular}

Mean (SD) are reported for all variables, except for diagnosis $(n)$, sex $(n-$ female $\left./ n_{\text {male }}\right)$ and education and Fazekas score (median [range]). For $\left[{ }^{18} \mathrm{~F}\right.$ ]flortaucipir $\mathrm{BP}_{\mathrm{ND}}$ and $R_{1}$, the range is additionally provided. Parametric $\left[{ }^{18} \mathrm{~F}\right]$ flortaucipir images were not partial volume-corrected. $M C I$ mild cognitive impairment, $A D$ Alzheimer's disease, $M M S E$ MiniMental State Examination, $B P_{N D}$ non-displaceable binding potential

\section{Associations between $\left[{ }^{18}\right.$ F]flortaucipir $\mathrm{BP}_{\mathrm{ND}}$ and $R_{1}$}

Higher $\left[{ }^{18} \mathrm{~F}\right]$ flortaucipir $\mathrm{BP}_{\mathrm{ND}}$ was associated with lower $R_{1}$ within the lateral temporal $(\mathrm{st} \beta-0.32[95 \% \mathrm{CI}-0.56$ to $0.08])$, parietal $(-0.43[-0.72$ to -0.14$])$ and occipital $(-0.53$ $[-0.78$ to -0.29$])$ ROI (Table 3$)$. Higher $\mathrm{BP}_{\mathrm{ND}}$ in the occipital ROI was also associated with lower $R_{1}$ in the parietal ROI $(-0.38[-0.64$ to -0.12$])$. All associations remained significant after FDR correction (Table 3). Figure 1 shows a selection of scatterplots for these associations. Addition of Fazekas scores to the model did not notably change the results (Supplementary Table 1).

\section{Voxel-wise associations with cognition}

Voxel-wise analyses (model 1) revealed that, in general, higher $\left[{ }^{18} \mathrm{~F}\right]$ flortaucipir $\mathrm{BP}_{\mathrm{ND}}$ was associated with worse $\operatorname{cog}$ nition (Fig. 2). More specifically, higher medial temporal $\mathrm{BP}_{\mathrm{ND}}$ associated with worse memory performance, and higher (orbito-)frontoparietal $\mathrm{BP}_{\mathrm{ND}}$ with worse scores on executive functioning (Fig. 2). Higher inferior temporal $\mathrm{BP}_{\mathrm{ND}}$ associated with worse language performance and higher (middle-)frontoparietal and occipital $\mathrm{BP}_{\mathrm{ND}}$ with worse attention scores (Fig. 2). After FWE correction, sparse associations with higher $\mathrm{BP}_{\mathrm{ND}}$ in the medial temporal regions and worse memory performance remained, as well as higher $\mathrm{BP}_{\mathrm{ND}}$ in the temporal (fusiform cortex) regions and worse language scores (data not shown). Associations between higher $\mathrm{BP}_{\mathrm{ND}}$ in the parietal and frontal regions and worse attention also survived FWE correction (data not shown).

Overall, lower $R_{1}$ associated with worse cognition (Fig. 2). In more detail, lower fronto-temporoparietal $R_{1}$ associated with worse scores on executive functioning and to a sparser extent with worse language performance (Fig. 2). Lower temporoparietal $R_{1}$ associated with worse attention scores (Fig. 2). None of the associations survived FWE correction (data not shown).

\section{Regional associations with cognition}

Regional linear regression analyses (model 1) revealed that higher medial temporal $\mathrm{BP}_{\mathrm{ND}}$ was associated with worse memory performance $(-0.43[-0.66$ to -0.20$])$, higher lateral temporal $\mathrm{BP}_{\mathrm{ND}}$ with worse scores on executive functioning $(-0.26[-0.52$ to -0.02$])$ and language $(-0.37$ [ -0.66 to $0.11])$, and higher parietal $\mathrm{BP}_{\mathrm{ND}}$ with worse executive functioning $(-0.46[-0.81$ to -0.23$])$, language $(-0.34[-0.76$ to $-0.03])$ and attention $(-0.50[-0.89$ to -0.25$])$ (Table 4; Fig. 3). Higher $\mathrm{BP}_{\mathrm{ND}}$ in the occipital ROI was associated with worse memory $(-0.27[-0.54$ to -0.00$])$, executive functioning $(-0.26[-0.53$ to -0.01$])$, language $(-0.40[-0.73$ to $0.14])$ and attention $(-0.37[-0.67$ to -0.10$])$ performance, and higher $\mathrm{BP}_{\mathrm{ND}}$ in the frontal ROI with worse executive functioning $(-0.34[-0.65$ to -0.13$])$ and attention $(-0.33$ $[-0.67$ to -0.08$])$. After FDR correction, the majority of significant associations remained (Table 4; Fig. 3).

Lower lateral temporal and parietal $R_{1}$ was associated with lower scores on executive functioning (0.27 [0.04 to 0.50$]$; 0.36 [0.15 to 0.60$])$, language ( 0.30 [0.04 to 0.57$] ; 0.28$ [0.02 to 0.57$])$ and attention $(0.33$ [0.08 to 0.57$] ; 0.48$ [0.26 to 0.71$]$ ) (Table 4; Fig. 3). Lower $R_{1}$ in the occipital ROI was associated with worse language $(0.28$ [0.03 to 0.57]) and attention (0.31 [0.07 to 0.59]) performance. By applying the FDR correction, the significant associations between lower $R_{l}$ in the parietal ROI and worse executive functioning remained, as well as the significant associations between lower $R_{1}$ in the lateral temporal, parietal and occipital ROI and worse attention scores (Table 4; Fig. 3). Scatterplots for a selection of these associations are presented in Fig. 1.

Finally, to examine the independent effects of tau pathology and $\mathrm{rCBF}$ on cognitive functioning, linear regression analyses including both $\left[{ }^{18} \mathrm{~F}\right]$ flortaucipir $\mathrm{BP}_{\mathrm{ND}}$ and $R_{1}$ were performed (model 2) (Table 4). Results revealed that higher medial temporal $\mathrm{BP}_{\mathrm{ND}}$ was independently associated with worse memory $(-0.44[-0.67$ to -0.20$])$, higher lateral temporal 


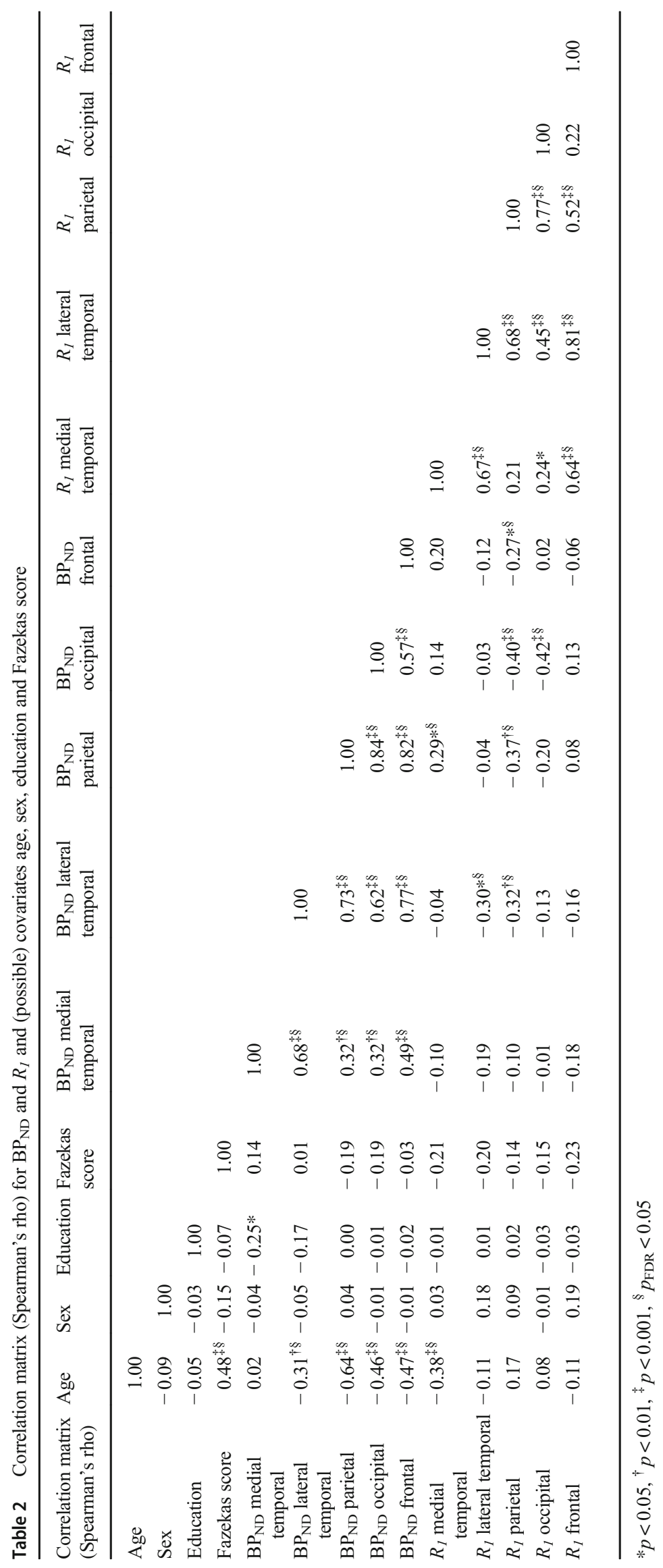


Table 3 Regional association between $\left[{ }^{18} \mathrm{~F}\right]$ flortaucipir $\mathrm{BP}_{\mathrm{ND}}$ (rows) and $R_{l}$ (columns)

\begin{tabular}{llllll}
\hline$\left[{ }^{18} \mathrm{~F}\right]$ flortaucipir $R_{I}$ & Medial temporal & Lateral temporal & Parietal & Occipital & Frontal \\
\hline$\left.{ }^{18} \mathrm{~F}\right]$ flortaucipir $\mathrm{BP}$ & & & & & \\
Medial temporal & $-0.10[-0.32-0.13]$ & $-0.21[-0.44-0.02]$ & $-0.11[-0.35-0.13]$ & $0.03[-0.21-0.27]$ & $-0.18[-0.41-0.05]$ \\
Lateral temporal & $-0.15[-0.38-0.08]$ & $-0.32^{*}[-0.56-0.08]$ & $-0.24[-0.49-0.01]$ & $-0.10[-0.35-0.16]$ & $-0.19[-0.43-0.06]$ \\
Parietal & $0.10[-0.18-0.38]$ & $-0.14[-0.44-0.16]$ & $-0.43^{*}[-0.72--0.14]$ & $-0.29[-0.59-0.00]$ & $0.04[-0.26-0.33]$ \\
Occipital & $0.02[-0.24-0.27]$ & $-0.07[-0.34-0.20]$ & $-0.38^{*}[-0.64--0.12]$ & $-0.53^{\dagger *}[-0.78--0.29]$ & $0.13[-0.13-0.40]$ \\
Frontal & $0.12[-0.12-0.36]$ & $-0.18[-0.43-0.08]$ & $-0.23[-0.49-0.03]$ & $0.09[-0.18-0.35]$ & $-0.14[-0.40-0.11]$ \\
\hline
\end{tabular}

Model is adjusted for age and sex. Standardized $\beta$ 's with $95 \%$ confidence intervals are reported. $B P_{N D}$ non-displaceable binding potential. $* p<0.01$, ${ }^{\dagger} p<0.001,{ }^{\ddagger} p_{\mathrm{FDR}}<0.05$

$\mathrm{BP}_{\mathrm{ND}}$ with worse language $(-0.31[-0.60$ to -0.04$])$ performance, and higher parietal $\mathrm{BP}_{\mathrm{ND}}$ with worse scores on executive functioning $(-0.36[-0.70$ to -0.11$])$ and attention $(-0.36[-0.72$ to -0.10$])$. Higher occipital $\mathrm{BP}_{\mathrm{ND}}$ was independently associated with worse language $(-0.33$ [0.68 to -0.03$])$ and higher frontal $\mathrm{BP}_{\mathrm{ND}}$ with lower scores on executive functioning $(-0.33[-0.64$ to -0.11$])$ and attention $(-0.31[-0.66$ to -0.06$])$. Most significant associations survived FDR correction (Table 4). For $\left[{ }^{18} \mathrm{~F}\right.$ ]flortaucipir $R_{1}$, lower values in the lateral temporal ROI were independently associated with worse attention ( 0.28 [0.03 to 0.54$]$ ), while low $R_{1}$ values in the parietal ROI were with lower scores on executive functioning $(0.27$ [0.06 to 0.50$])$ and attention (0.39 [0.17 to 0.62]). After FDR correction, the association between lower $R_{1}$ in the parietal ROI and worse attention remained (Table 4).
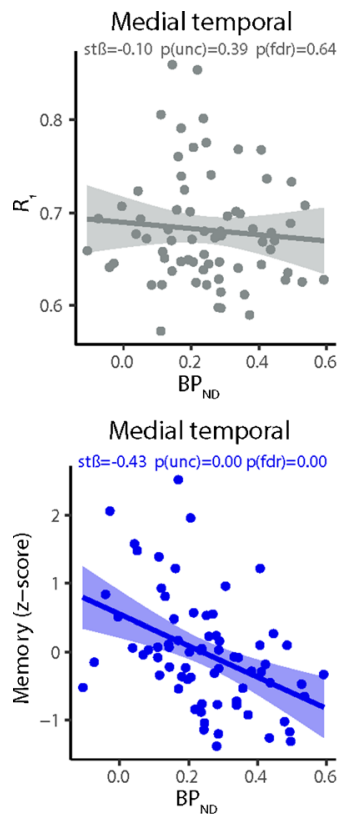

Medial temporal
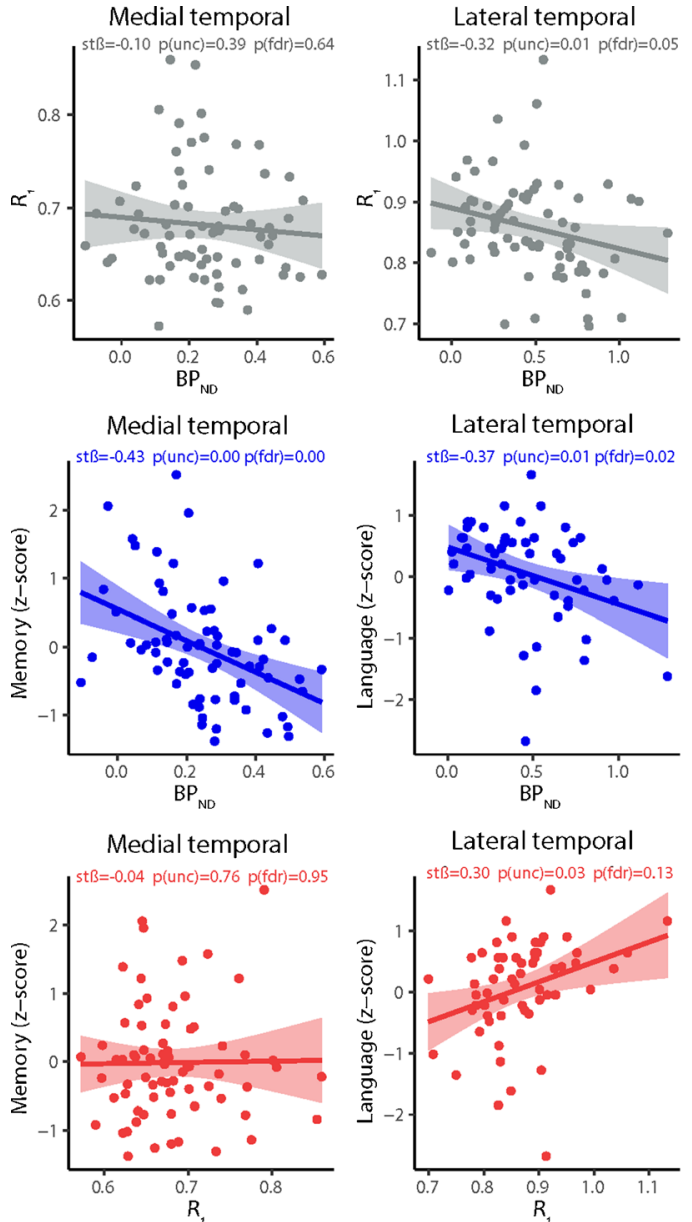

Lateral temporal

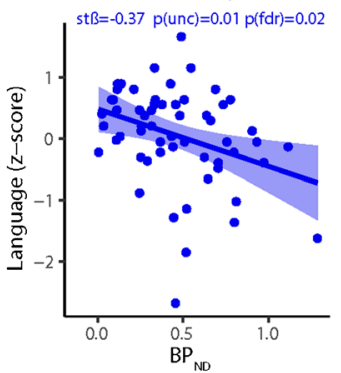

Lateral temporal
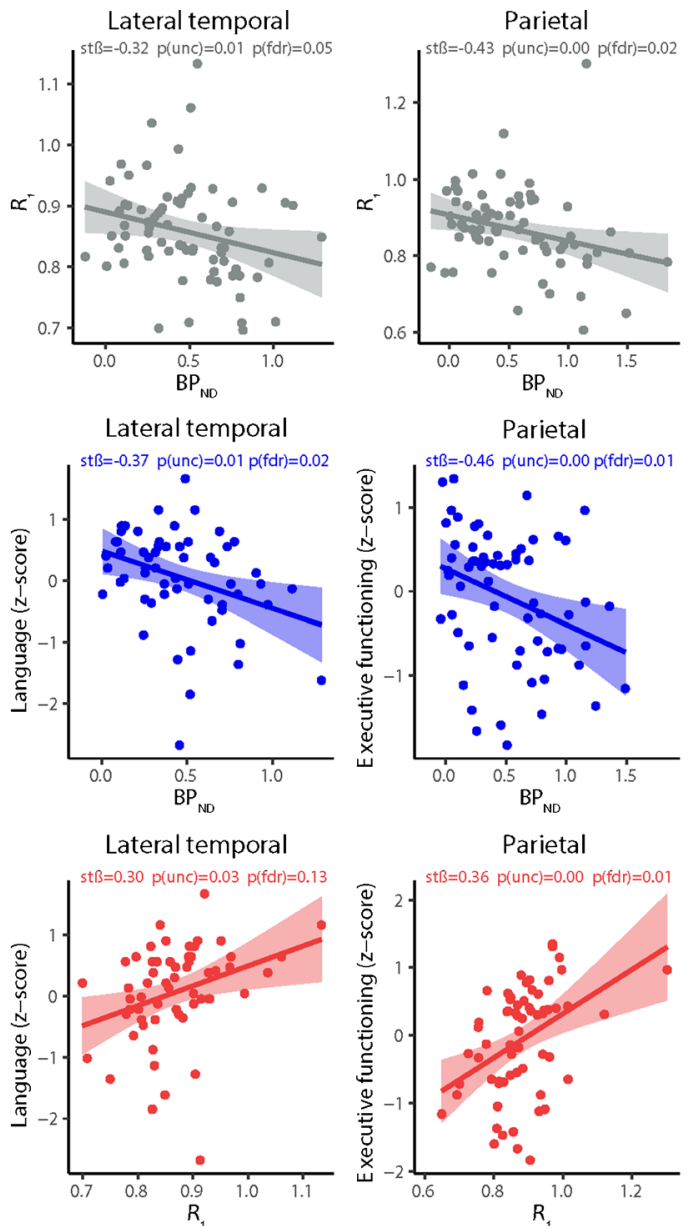

Parietal

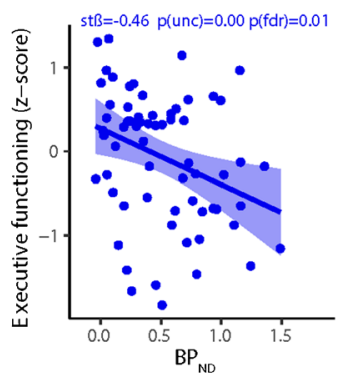

Parietal

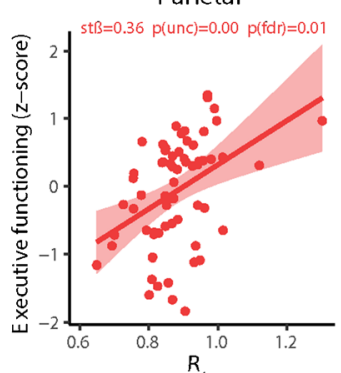

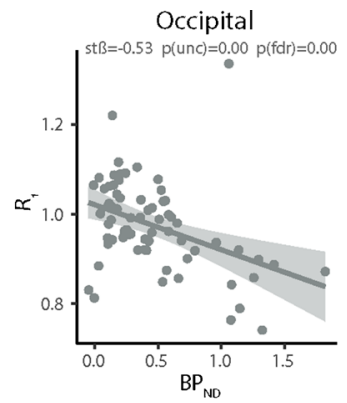
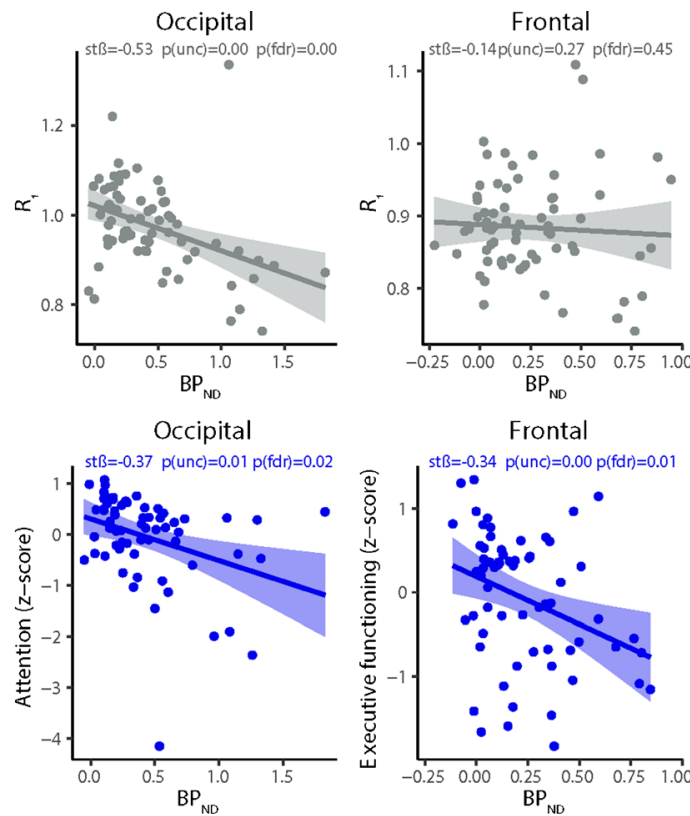

Occipital

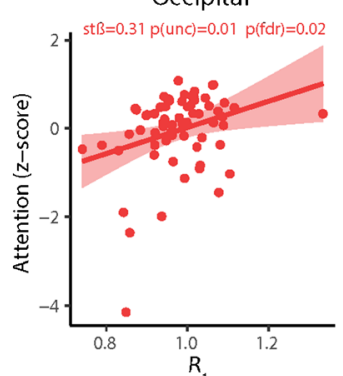

Frontal

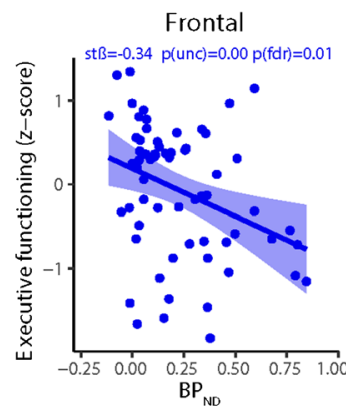

Frontal

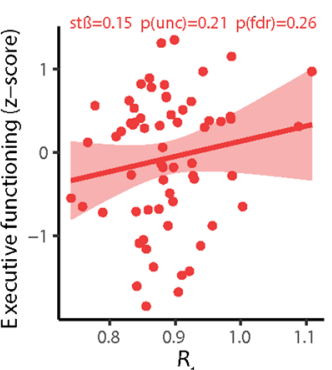

Fig. 1 Selection of scatterplots between $\left[{ }^{18} \mathrm{~F}\right]$ flortaucipir $\mathrm{BP}_{\mathrm{ND}}$ and/or $R_{l}$ and/or cognition. $B P_{N D}$ non-displaceable binding potential, st $\beta$ standardized $\beta, p(u n c)$ uncorrected $p$ value, $p(f d r) p$ value corrected for multiple comparisons using the Benjamini-Hochberg procedure with a false discovery rate (FDR) Q value of 5\%. A $p$ value below 0.05 was considered statistically significant 
Fig. 2 Voxel-wise associations between $\left[{ }^{18} \mathrm{~F}\right]$ flortaucipir $\mathrm{BP}_{\mathrm{ND}}$ or $R_{I}$ and cognition. Voxel-wise regression analyses were pereducation. Voxels with a significant $(p<0.001$, uncorrected) association are displayed. $B P_{N D}$ non-displaceable binding potential, $A$ anterior, $P$ posterior, $R$ right, $L$ left formed, adjusted for age, sex and
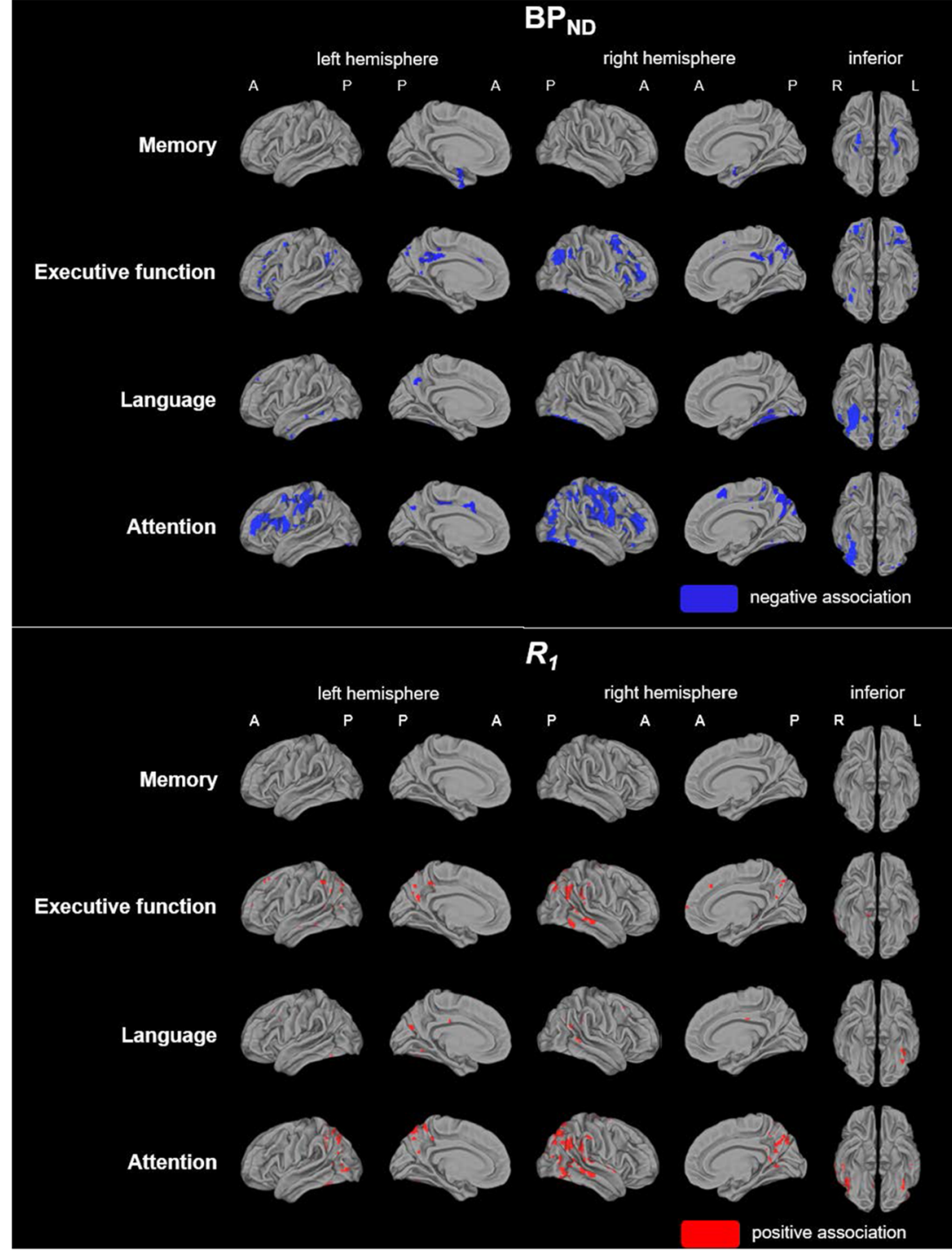

Tau pathology and $\mathrm{rCBF}$ are independently associated with cognition in AD

Overall, partial volume-corrected data yielded slightly higher values for both $\left[{ }^{18} \mathrm{~F}\right.$ ]flortaucipir $\mathrm{BP}_{\mathrm{ND}}$ and $R_{l}$ (Supplementary Table 2), but results from regression analyses remained essentially comparable (Supplementary Tables 3 \& 4; Supplementary FIGs. 1 \& 2).

\section{Discussion}

The present study used a single dynamic $\left[{ }^{18} \mathrm{~F}\right]$ flortaucipir PET scan to examine the relationship between tau pathology, $\mathrm{rCBF}$ and cognition in AD. The main finding is that high levels of tau pathology and low levels of rCBF were independently associated with worse cognitive performance across various domains.
An important finding in the present study is that tau pathology and $\mathrm{rCBF}$, at least in part, independently contribute to cognitive deficits in AD. A previous study demonstrated that tau pathology was also independently associated with specific cognitive impairment in AD in the context of neurodegeneration [29]. This leads to the notion that tau pathology may impact cognitive performance directly, but also indirectly through a variety of mechanisms [29]. One such mechanisms might be rCBF, since some of the associations found between $\left[{ }^{18} \mathrm{~F}\right]$ flortaucipir $\mathrm{BP}_{\mathrm{ND}}$ and cognition in the present study disappeared when $R_{1}$ was included in the model simultaneously. Other factors possibly explaining the tau pathologyindependent associations between $\mathrm{rCBF}$ and cognition in AD might be the presence of other down- or upstream pathological factors like tau-independent atrophy, vascular 
Table 4 Regional associations between $\left[{ }^{18} \mathrm{~F}\right]$ flortaucipir $\mathrm{BP}_{\mathrm{ND}}$ and $R_{I}$ and cognition

\begin{tabular}{|c|c|c|c|c|c|}
\hline & & Memory $(n=71)$ & Executive functioning $(n=64)$ & Language $(n=59)$ & Attention $(n=64)$ \\
\hline \multicolumn{6}{|l|}{ Model 1} \\
\hline \multirow[t]{2}{*}{ Medial temporal } & $\mathrm{BP}_{\mathrm{ND}}$ & $-0.43^{\ddagger \delta}[-0.66--0.20]$ & $-0.08[-0.34-0.16]$ & $-0.17[-0.48-0.12]$ & $0.00[-0.28-0.28]$ \\
\hline & $R_{1}$ & $-0.04[-0.30-0.22]$ & $0.03[-0.22-0.28]$ & $0.10[-0.18-0.38]$ & $0.07[-0.21-0.35]$ \\
\hline \multirow[t]{2}{*}{ Lateral temporal } & $\mathrm{BP}_{\mathrm{ND}}$ & $-0.22[-0.48-0.04]$ & $-0.26^{*}[-0.52--0.02]$ & $-0.37^{\dagger \S}[-0.66--0.11]$ & $-0.25[-0.54-0.02]$ \\
\hline & $R_{1}$ & $0.03[-0.22-0.27]$ & $0.27 *[0.04-0.50]$ & $0.30 *[0.04-0.57]$ & $0.33^{\dagger \S}[0.08-0.57]$ \\
\hline \multirow[t]{2}{*}{ Parietal } & $\mathrm{BP}_{\mathrm{ND}}$ & $-0.23[-0.52-0.07]$ & $-0.46^{\dagger \S}[-0.81--0.23]$ & $-0.34 *[-0.76--0.03]$ & $-0.50^{\dagger \S}[-0.89--0.25]$ \\
\hline & $R_{l}$ & $0.06[-0.18-0.30]$ & $0.36^{\dagger \S}[0.15-0.60]$ & $0.28 *[0.02-0.57]$ & $0.48^{\ddagger \S}[0.26-0.71]$ \\
\hline \multirow[t]{2}{*}{ Occipital } & $\mathrm{BP}_{\mathrm{ND}}$ & $-0.27 *[-0.54--0.00]$ & $-0.26^{*}[-0.53--0.01]$ & $-0.40^{\dagger \S}[-0.73--0.14]$ & $-0.37^{\dagger \S}[-0.67--0.10]$ \\
\hline & $R_{1}$ & $0.13[-0.12-0.37]$ & $0.17[-0.06-0.43]$ & $0.28 *[0.03-0.57]$ & $0.31 * \S[0.07-0.59]$ \\
\hline \multirow[t]{2}{*}{ Frontal } & $\mathrm{BP}_{\mathrm{ND}}$ & $-0.14[-0.40-0.12]$ & $-0.34^{\dagger \S}[-0.65--0.13]$ & $-0.21[-0.57-0.09]$ & $-0.33 * \S[-0.67--0.08]$ \\
\hline & $R_{1}$ & $-0.12[-0.37-0.13]$ & $0.15[-0.09-0.40]$ & $0.13[-0.15-0.41]$ & $0.13[-0.13-0.40]$ \\
\hline \multicolumn{6}{|l|}{ Model 2} \\
\hline \multirow[t]{2}{*}{ Medial temporal } & $\mathrm{BP}_{\mathrm{ND}}$ & $-0.44^{\ddagger \S}[-0.67--0.20]$ & $-0.08[-0.34-0.17]$ & $-0.17[-0.48-0.12]$ & $0.01[-0.28-0.29]$ \\
\hline & $R_{1}$ & $-0.09[-0.33-0.15]$ & $0.03[-0.23-0.28]$ & $0.10[0.19-0.38]$ & $0.07[-0.21-0.35]$ \\
\hline \multirow[t]{2}{*}{ Lateral temporal } & $\mathrm{BP}_{\mathrm{ND}}$ & $-0.23[-0.51-0.04]$ & $-0.19[-0.46-0.05]$ & $-0.31 *[-0.60--0.04]$ & $-0.16[-0.45-0.11]$ \\
\hline & $R_{1}$ & $-0.05[-0.30-0.21]$ & $0.21[-0.02-0.46]$ & $0.22[-0.04-0.49]$ & $0.28 *[0.03-0.54]$ \\
\hline \multirow[t]{2}{*}{ Parietal } & $\mathrm{BP}_{\mathrm{ND}}$ & $-0.23[-0.55-0.09]$ & $-0.36^{\dagger \S}[-0.70--0.11]$ & $-0.27[-0.68-0.05]$ & $-0.36 *[-0.72--0.10]$ \\
\hline & $R_{1}$ & $-0.00[-0.26-0.25]$ & $0.27^{*}[0.06-0.50]$ & $0.22[-0.05-0.52]$ & $0.39^{\dagger \S}[0.17-0.62]$ \\
\hline \multirow[t]{2}{*}{ Occipital } & $\mathrm{BP}_{\mathrm{ND}}$ & $-0.26[-0.57-0.04]$ & $-0.21[-0.52-0.07]$ & $-0.33 *[-0.68--0.03]$ & $-0.27[-0.59-0.03]$ \\
\hline & $R_{1}$ & $0.02[-0.25-0.28]$ & $0.09[-0.17-0.37]$ & $0.15[-0.13-0.46]$ & $0.20[-0.07-0.50]$ \\
\hline \multirow[t]{2}{*}{ Frontal } & $\mathrm{BP}_{\mathrm{ND}}$ & $-0.16[-0.42-0.11]$ & $-0.33^{\dagger \S}[-0.64--0.11]$ & $-0.20[-0.56-0.10]$ & $-0.31 * \S[-0.66--0.06]$ \\
\hline & $R_{1}$ & $-0.14[-0.39-0.11]$ & $0.11[-0.12-0.34]$ & $0.11[-0.16-0.39]$ & $0.09[-0.17-0.35]$ \\
\hline
\end{tabular}

Models are adjusted for age, sex and education. $\left[{ }^{18} \mathrm{~F}\right]$ Flortaucipir $\mathrm{BP}_{\mathrm{ND}}$ and $R_{l}$ were included in the model separately (model 1$)$ and simultaneously (model 2). Standardized $\beta$ 's with $95 \%$ confidence intervals are reported. $B P_{N D}$ non-displaceable binding potential. $*^{*} p<0.05,{ }^{\dagger} p<0.01,{ }^{\ddagger} p<0.001$, ${ }^{\S} p_{\mathrm{FDR}}<0.05$

pathology or other proteinopathies. Vascular damage for example might lead to impaired $\mathrm{rCBF}$, possibly causing an increase in amyloid- $\beta$ accumulation, which in turn can lead to inflammation and neuronal dysfunction, leading to cognitive deficits [30]. Further research is required, however, to gain knowledge about the mechanisms explaining the tauindependent relationships between $\mathrm{rCBF}$ and cognition in AD.

\section{Associations between tau pathology, rCBF and cognition}

Strong (regional) associations between tau pathology and cognitive deficits in $\mathrm{AD}$ have been established by multiple (imaging) studies [10-12,31], and results of the present study are generally in line with previous findings. As expected, tau pathology in the medial temporal regions showed strong associations with memory, while tau pathology in temporoparietal regions was associated with language. High levels of tau pathology in frontal regions were associated with more anteriorly based cognitive functions, like executive function and attention.
Although the association between $\mathrm{CBF}$ and cognition in $\mathrm{AD}$ has not been studied using this $\left[{ }^{18} \mathrm{~F}\right]$ flortaucipir PET approach before, other studies investigated these associations by using [ $\left.{ }^{18} \mathrm{~F}\right] \mathrm{FDG}$ PET or MRI techniques (such as arterial spin labeling (ASL)) to measure (proxies of) $\mathrm{CBF}[13,32]$. These studies demonstrated that in $\mathrm{AD}$, reduced $\mathrm{CBF}$ is generally associated with worse global cognition [13, 32] and not with domain-specific cognitive impairments (memory, executive function, language, attention and visuospatial functioning) [13]. Nonetheless, it was also demonstrated that most associations with cognition were found for low CBF in parietal and occipital regions, while least associations were found for temporal and frontal CBF [13]. This is in line with our results, although we also found multiple associations between cognition and low CBF in lateral temporal regions. This might be explained by the fact that the former study used a ROI covering the entire temporal cortex and did not differentiate between medial and lateral temporal regions. Both studies used subjects from the Amsterdam Dementia Cohort and used a similar approach to assess cognition, but another striking similarity between the former and present study is the range of regression coefficients for significant associations between 

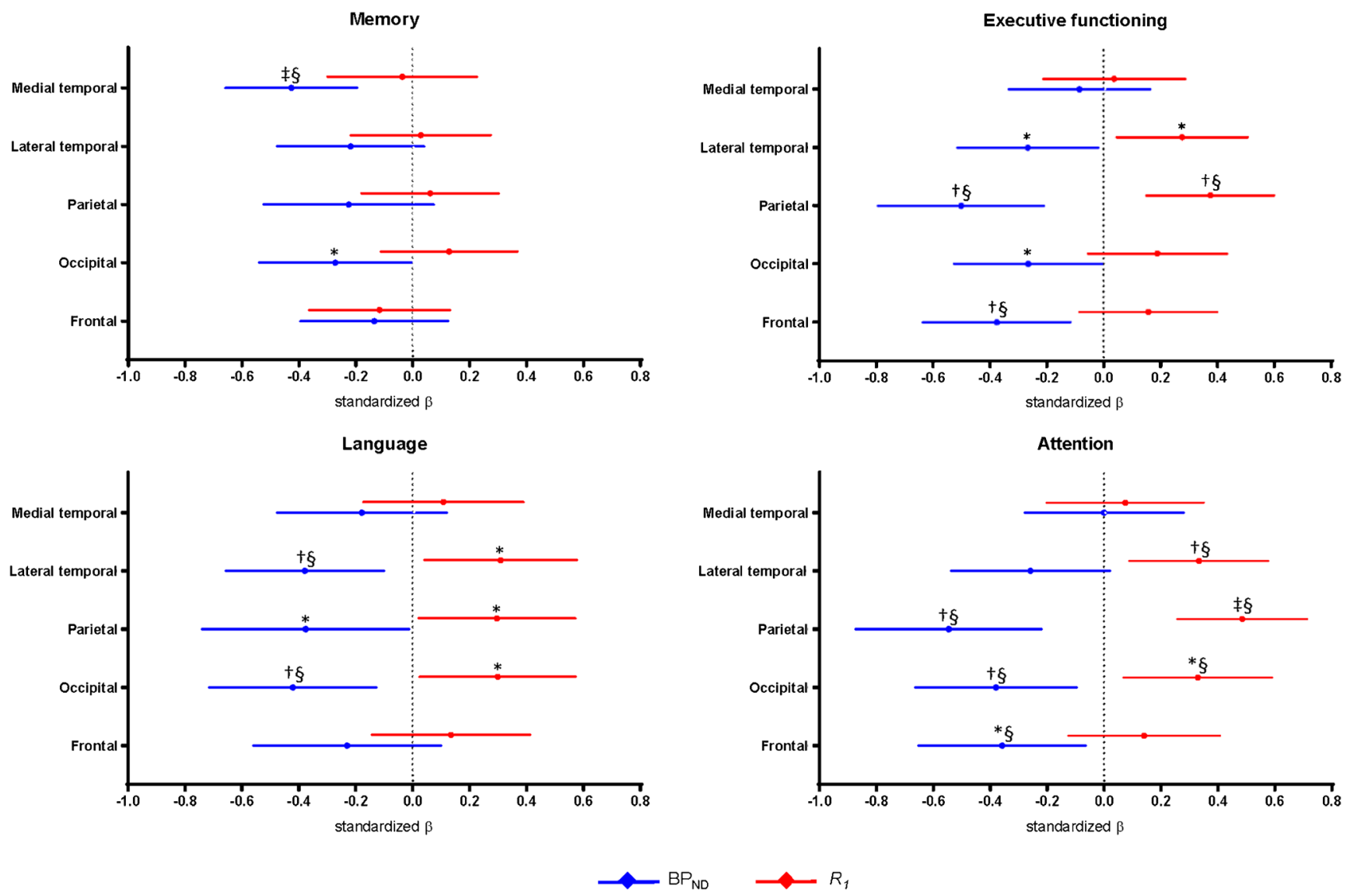

Fig. 3 Regional associations between $\left[{ }^{18} \mathrm{~F}\right]$ flortaucipir $\mathrm{BP}_{\mathrm{ND}}$ or $R_{l}$ and cognition. $\left[{ }^{18} \mathrm{~F}\right]$ Flortaucipir $\mathrm{BP}_{\mathrm{ND}}$ and $R_{l}$ were included in the model separately (model 1). Displayed are regression estimates (standardized

$\beta$ 's) with $95 \%$ confidence intervals. All analyses are adjusted for age, sex and education. $B P_{N D}$ non-displaceable binding potential. ${ }^{*} p<0.05$, ${ }^{\dagger} p<0.01,{ }^{\star} p<0.001,{ }^{8} p_{\text {FDR }}<0.05$

$\mathrm{CBF}$ and cognition. Standardized regression coefficients ranged from 0.22 till 0.42 across the cognitive domains in the former study [13], and ranged from 0.27 till 0.48 in the present study, indicating comparable effect sizes.

\section{Regional association between tau pathology and low rCBF}

Relative CBF is tightly correlated with measures of metabolic activity such as $\left[{ }^{18} \mathrm{~F}\right] \mathrm{FDG}$ PET $[4,5,9]$. Earlier studies investigating $\left[{ }^{18} \mathrm{~F}\right]$ flortaucipir and $\left[{ }^{18} \mathrm{~F}\right] \mathrm{FDG}$ PET in AD found considerable overlap between higher levels of tau tracer uptake and lower levels of metabolic activity $[11,33]$, with moderate correlation coefficients across 30 predefined brain regions. The present study used $R_{1}$ as proxy for $\mathrm{rCBF}$, and in line with the previously described study [33], we also found spatial overlap between high levels of tau pathology and low rCBF, with comparable standardized regression coefficients. The overlap of high levels of tau pathology and low levels of rCBF was in both studies not completely uniform across all brain regions, suggesting that both measures represent complementary aspects of AD pathology [33]. A potential explanation might be that tau pathology may develop prior to or

even (partially) drive impaired metabolic activity or CBF, creating a time-lag between both pathological mechanism leading to topographical differences [34]. Alternatively, other pathological processes besides tau pathology may contribute to impaired metabolic activity or $\mathrm{CBF}$, such as other proteinopathies. Vascular pathology has been linked to $\mathrm{AD}$ [35] and might have an impact on for example rCBF. However, in our study, the influence of vascular pathology showed to be negligible, since no correlation between Fazekas score and tau pathology or $\mathrm{rCBF}$ was found, and addition of Fazekas score to the regression model assessing the association between tau pathology and $\mathrm{rCBF}$ did not notably change results.

\section{Strengths and limitations}

This study has several strengths, including the use of $\left[{ }^{18} \mathrm{~F}\right]$ flortaucipir $R_{l}$ as a measure of $\mathrm{rCBF}$, since this tracer has not been used in this context before, while $\left[{ }^{18} \mathrm{~F}\right]$ flortaucipir currently is the most widely used tracer for tau pathology in AD. Another strength is that both measures were derived from a single dynamic $\left[{ }^{18} \mathrm{~F}\right]$ flortaucipir PET scan, thereby circumventing the need for a dual-tracer study and avoiding 
bias caused by time-lags between measures of tau pathology and $\mathrm{rCBF}$. Furthermore, analyses were repeated with partial volume-corrected data, and results remained essentially comparable; hence, we feel that our findings are not biased by atrophy to a large extent.

This study also has some limitations. The $\mathrm{AD}$ patients in this study were relatively young, which might hamper generalizability of results to older patient populations. Also, because our sample included only ten 'MCI due to AD' patients, further research is needed to elucidate potential differences in the $\mathrm{BP}_{\mathrm{ND}}-R_{1}$ relationship between diagnostic groups. Furthermore, it might be difficult to draw firm conclusions about the performance of $\left[{ }^{18} \mathrm{~F}\right]$ flortaucipir $R_{l}$ compared to other measures of $\mathrm{CBF}$ due to the lack of a golden standard for measuring CBF. At last, this study has a cross-sectional design, which excluded the possibility to investigate whether the associations found between tau pathology, $\mathrm{rCBF}$ and cognition in AD represent causality. Therefore, longitudinal designs are required.

\section{Conclusion}

This study demonstrates that tau pathology and rCBF derived from a single dynamic $\left[{ }^{18} \mathrm{~F}\right]$ flortaucipir PET scan are associated in a region-specific matter, with high levels of tau pathology being generally present in areas with low levels of rCBF. Lower cognitive scores are associated with higher levels of tau pathology and lower levels of rCBF. A substantial amount of these associations remained present when correcting for the other PET measure, indicating that tau pathology and $\mathrm{rCBF}$ (at least in part) independently contribute to cognitive deficits in AD. Besides, this study indicates that the use of dynamic $\left[{ }^{18} \mathrm{~F}\right]$ flortaucipir PET might sometimes be preferable, since accurate quantification of tau pathology and an additional functional measure like $\mathrm{rCBF}$ can be derived from a single scan.

Acknowledgements We kindly thank all participants for their contribution. Research of Amsterdam Alzheimer Center is part of the Neurodegeneration program of Amsterdam Neuroscience. The Amsterdam Alzheimer Center is supported by Alzheimer Nederland and Stichting VUmc funds. $\left[{ }^{18} \mathrm{~F}\right]$ Flortaucipir PET scans were made possible by Avid Radiopharmaceuticals Inc.

Funding information This study was funded by a ZonMW Memorabel grant.

\section{Compliance with ethical standards}

Conflict of interest Visser, Wolters, Verfaillie, Coomans, Timmers, Tuncel, Reimand, Boellaard, Windhorst and Ossenkoppele declare that they have no conflict of interest.

Van der Flier received grant support from ZonMW, NWO, EU-FP7, Alzheimer Nederland, CardioVascular Onderzoek Nederland, Stichting
Dioraphte, Gieskes-Strijbis Fonds, Boehringer Ingelheim, Piramal Neuroimaging, Roche BV, Janssen Stellar, Combinostics. All funding is paid to the institution. WvdF holds the Pasman chair.

Van Berckel received research support from ZON-MW, AVID radiopharmaceuticals, CTMM and Janssen Pharmaceuticals. He is a trainer for Piramal and GE. He receives no personal honoraria.

Scheltens received grant support (to the institution) from GE Healthcare, Danone Research, Piramal and MERCK. In the past 2 years, he has received consultancy/speaker fees from Lilly, GE Healthcare, Novartis, Forum, Sanofi, Nutricia, Probiodrug and EIP Pharma. All funding is paid to the institution.

No other potential conflicts of interest relevant to this article exist.

Ethical approval All procedures performed in studies involving human participants were in accordance with the ethical standards of the institutional and/or national research committee and with the 1964 Helsinki Declaration and its later amendments or comparable ethical standards.

Informed consent Informed consent was obtained from all individual participants included in the study.

Open Access This article is licensed under a Creative Commons Attribution 4.0 International License, which permits use, sharing, adaptation, distribution and reproduction in any medium or format, as long as you give appropriate credit to the original author(s) and the source, provide a link to the Creative Commons licence, and indicate if changes were made. The images or other third party material in this article are included in the article's Creative Commons licence, unless indicated otherwise in a credit line to the material. If material is not included in the article's Creative Commons licence and your intended use is not permitted by statutory regulation or exceeds the permitted use, you will need to obtain permission directly from the copyright holder. To view a copy of this licence, visit http://creativecommons.org/licenses/by/4.0/.

\section{References}

1. Schöll M, Maass A, Mattsson N, Ashton N, Blennow K, Zetterberg H, et al. Biomarkers for tau pathology. Mol Cell Neurosci 2019;97: 18-33. https://doi.org/10.1016/j.men.2018.12.001

2. Ossenkoppele R, Prins N, Berckel B. Amyloid imaging in clinical trials. Alzheimers Res Ther. 2013;5:36. https://doi.org/10.1186/ alzrt195.

3. Yaqub M, Tolboom N, Boellaard R, van Berckel BN, van Tilburg EW, Luurtsema G, et al. Simplified parametric methods for [11C] PIB studies. Neuroimage. 2008;42:76-86.

4. Peretti DE, García DV, Reesink FE, van der Goot T, De Deyn PP, de Jong BM, et al. Relative cerebral flow from dynamic PIB scans as an alternative for FDG scans in Alzheimer's disease PET studies. PLoS One. 2019;14:e0211000.

5. Rodriguez-Vieitez E, Leuzy A, Chiotis K, Saint-Aubert L, Wall A, Nordberg A. Comparability of [18F] THK5317 and [11C] PIB blood flow proxy images with [18F] FDG positron emission tomography in Alzheimer's disease. J Cereb Blood Flow Metab. 2017;37:740-9.

6. Peretti DE, García DV, Reesink FE, Doorduin J, de Jong BM, De Deyn PP, et al. Diagnostic performance of regional cerebral blood flow images derived from dynamic PIB scans in Alzheimer's disease. EJNMMI Res. 2019;9:59.

7. Joseph-Mathurin N, Su Y, Blazey TM, Jasielec M, Vlassenko A, Friedrichsen K, et al. Utility of perfusion PET measures to assess neuronal injury in Alzheimer's disease. Alzheimers Dement. 2018;10:669-77. 
8. Chen YJ, Rosario BL, Mowrey W, Laymon CM, Lu X, Lopez OL, et al. Relative $11 \mathrm{C}-\mathrm{PiB}$ delivery as a proxy of relative $\mathrm{CBF}$ : quantitative evaluation using single-session $15 \mathrm{O}$-water and $11 \mathrm{C}-\mathrm{PiB}$ PET. J Nucl Med. 2015;56:1199-205.

9. Ottoy J, Verhaeghe J, Niemantsverdriet E, De Roeck E, Wyffels L, Ceyssens S, et al. 18F-FDG PET, the early phases and the delivery rate of $18 \mathrm{~F}-\mathrm{AV} 45 \mathrm{PET}$ as proxies of cerebral blood flow in Alzheimer's disease: validation against 15O-H2O PET. Alzheimers Dement. 2019. https://doi.org/10.1016/j.jalz.2019.05. 010 .

10. Johnson KA, Schultz A, Betensky RA, Becker JA, Sepulcre J, Rentz D, et al. Tau positron emission tomographic imaging in aging and early Alzheimer disease. Ann Neurol. 2016;79:110-9.

11. Ossenkoppele R, Schonhaut DR, Schöll M, Lockhart SN, Ayakta N, Baker SL, et al. Tau PET patterns mirror clinical and neuroanatomical variability in Alzheimer's disease. Brain. 2016;139:155167.

12. Pontecorvo MJ, Devous MD Sr, Navitsky M, Lu M, Salloway S, Schaerf FW, et al. Relationships between flortaucipir PET tau binding and amyloid burden, clinical diagnosis, age and cognition. Brain. 2017;140:748-63.

13. Leeuwis AE, Benedictus MR, Kuijer JP, Binnewijzend MA, Hooghiemstra AM, Verfaillie SC, et al. Lower cerebral blood flow is associated with impairment in multiple cognitive domains in Alzheimer's disease. Alzheimers Dement. 2017;13:531-40.

14. Van Der Flier WM, Scheltens P. Amsterdam dementia cohort: performing research to optimize care. J Alzheimers Dis. 2018;62: 1091-111.

15. McKhann GM, Knopman DS, Chertkow H, Hyman BT, Jack CR $\mathrm{Jr}$, Kawas CH, et al. The diagnosis of dementia due to Alzheimer's disease: recommendations from the National Institute on AgingAlzheimer's association workgroups on diagnostic guidelines for Alzheimer's disease. Alzheimers Dement. 2011;7:263-9.

16. Albert MS, DeKosky ST, Dickson D, Dubois B, Feldman HH, Fox $\mathrm{NC}$, et al. The diagnosis of mild cognitive impairment due to Alzheimer's disease: recommendations from the National Institute on Aging-Alzheimer's Association workgroups on diagnostic guidelines for Alzheimer's disease. Alzheimers Dement. 2011;7: $270-9$.

17. Tijms BM, Willemse EA, Zwan MD, Mulder SD, Visser PJ, van Berckel BN, et al. Unbiased approach to counteract upward drift in cerebrospinal fluid amyloid- $\beta$ 1-42 analysis results. Clin Chem. 2018;64:576-85.

18. Jack CR Jr, Bennett DA, Blennow K, Carrillo MC, Dunn B, Haeberlein SB, et al. NIA-AA research framework: toward a biological definition of Alzheimer's disease. Alzheimers Dement. 2018;14:535-62.

19. Golla SS, Timmers T, Ossenkoppele R, Groot C, Verfaillie S, Scheltens P, et al. Quantification of tau load using [18 F] AV1451 PET. Mol Imaging Biol. 2017;19:963-71.

20. Hammers A, Allom R, Koepp MJ, Free SL, Myers R, Lemieux L, et al. Three-dimensional maximum probability atlas of the human brain, with particular reference to the temporal lobe. Hum Brain Mapp. 2003;19:224-47.

21. Gunn RN, Lammertsma AA, Hume SP, Cunningham VJ. Parametric imaging of ligand-receptor binding in PET using a simplified reference region model. Neuroimage. 1997;6:279-87.
22. Lammertsma AA. Forward to the past: the case for quantitative PET imaging. J Nucl Med. 2017;58:1019-24.

23. Golla SS, Wolters EE, Timmers T, Ossenkoppele R, van der Weijden CW, Scheltens P, et al. Parametric methods for $[18 \mathrm{~F}]$ flortaucipir PET. J Cereb Blood Flow Metab. 2019;0271678X18820765. 40(2):365-373. https://doi.org/10. $1177 / 0271678 X 18820765$

24. Timmers T, Ossenkoppele R, Visser D, Tuncel H, Wolters E, Verfaillie SCJ, et al. Test-retest repeatability of $[18 \mathrm{~F}]$ Flortaucipir PET in Alzheimer's disease and cognitively normal individuals. J Cereb Blood Flow Metab. 2019; in press.https://doi.org/10.1177/ $0271678 X 19879226$

25. Golla SS, Lubberink M, van Berckel BN, Lammertsma AA, Boellaard R. Partial volume correction of brain PET studies using iterative deconvolution in combination with HYPR denoising. EJNMMI Res. 2017;7:36.

26. Cysouw M, Golla S, Frings V, Smit E, Hoekstra O, Kramer G, et al. Partial-volume correction in dynamic PET-CT: effect on tumor kinetic parameter estimation and validation of simplified metrics. EJNMMI Res. 2019;9:12.

27. Fazekas F, Chawluk JB, Alavi A, Hurtig HI, Zimmerman RA. MR signal abnormalities at $1.5 \mathrm{~T}$ in Alzheimer's dementia and normal aging. Am J Roentgenol. 1987;149:351-6.

28. Groot C, van Loenhoud AC, Barkhof F, van Berckel BN, Koene T, Teunissen CC, et al. Differential effects of cognitive reserve and brain reserve on cognition in Alzheimer disease. Neurology. 2018;90:e149-e56.

29. Bejanin A, Schonhaut DR, La Joie R, Kramer JH, Baker SL, Sosa $\mathrm{N}$, et al. Tau pathology and neurodegeneration contribute to cognitive impairment in Alzheimer's disease. Brain. 2017;140:3286300.

30. Kisler K, Nelson AR, Montagne A, Zlokovic BV. Cerebral blood flow regulation and neurovascular dysfunction in Alzheimer disease. Nat Rev Neurosci. 2017;18:419. https://doi.org/10.1038/nrn. 2017.48

31. Digma LA, Madsen JR, Reas ET, Dale AM, Brewer JB, Banks SJ. Tau and atrophy: domain-specific relationships with cognition. Alzheimers Res Ther. 2019;11:65.

32. Landau SM, Harvey D, Madison CM, Koeppe RA, Reiman EM, Foster NL, et al. Associations between cognitive, functional, and FDG-PET measures of decline in AD and MCI. Neurobiol Aging. 2011;32:1207-18.

33. Dronse J, Fliessbach K, Bischof GN, von Reutern B, Faber J, Hammes $J$, et al. In vivo patterns of tau pathology, amyloid- $\beta$ burden, and neuronal dysfunction in clinical variants of Alzheimer's disease. J Alzheimers Dis. 2017;55:465-71.

34. Jack CR Jr, Knopman DS, Jagust WJ, Petersen RC, Weiner MW, Aisen PS, et al. Tracking pathophysiological processes in Alzheimer's disease: an updated hypothetical model of dynamic biomarkers. Lancet Neurol. 2013;12:207-16.

35. Altman R, Rutledge JC. The vascular contribution to Alzheimer's disease. Clin Sci. 2010;119:407-21.

Publisher's note Springer Nature remains neutral with regard to jurisdictional claims in published maps and institutional affiliations. 